\title{
La atención al paciente crónico complejo
}

\author{
Clinical care of the chronic complex patient
}

\section{Pilar Román Sánchez}

Vicepresidente de la Sociedad Española de Medicina Interna (SEMI)

Presidente del Comité Científico del IV Congreso para la Atención al Paciente Crónico.

No descubro nada a los profesionales sanitarios de cualquier ámbito si afirmo que el patrón de enfermedades ha cambiado en los últimos 30 años. Todos somos conscientes de que hemos pasado de la atención puntual a procesos agudos, principalmente de naturaleza infecciosa, a la atención repetida de episodios de descompensación de patologías crónicas en personas de edad avanzada. Pero ver plasmada y cuantificada esta realidad, como hacen los doctores Montes y Casariego en la presentación de esta edición especial de Galicia Clínica, es, por decirlo de forma suave, altamente preocupante. Y lo era ya en años previos cuando la situación económica de nuestro país era próspera. Bien es cierto que durante esos pasados años de bonanza, los sistemas regionales de salud han ido acumulando deuda de manera continuada y que a este hecho se añade, con la situación de crisis económica actual, una caída precipitada de los ingresos del Estado y de la Comunidades Autónomas, lo que provoca que la deuda llegue casi a transformarse en una quiebra formal, situación nueva e inquietante.

Todos sabemos que nuestro sistema sanitario, como la mayoría de los de nuestro entorno, sigue enfocado a la atención de episodios agudos. Que los profesionales "esperamos" a que el paciente, generalmente poco informado de sus enfermedades, demande asistencia cuando siente que lo necesita y que esa necesidad ha ido aumentando a lo largo de los años siguiendo comportamientos sociales, a veces ajenos a la propia enfermedad. Entonces, en 10 minutos en el caso de Atención primaria o en 20 en el de Especializada, debemos realizar la anamnesis, exploración, el diagnóstico de presunción, hablar con el paciente y su familia, pedir exploraciones complementarias y realizar la prescripción de fármacos si proceden o, en caso contrario, explicar que no procede prescripción de pruebas o fármaco alguno, situación ésta que consume mucho más tiempo que hacer lo contrario en una sociedad totalmente "medicalizada" como es la nuestra, hecho al que los profesionales sanitarios hemos contribuido. Y, generalmente, prescribimos pruebas o fármacos porque es más cómodo y el paciente así lo demanda. Y, muchas veces, remitimos al paciente a otro profesional más especializado, también porque es más cómodo, seguro para nosotros y porque el paciente así lo demanda. Este especialista actuará de la misma forma, sin conexión con el resto de profesionales que han atendido al paciente y con la visión parcial de su especialidad. El resultado será la multiplicidad y repetición de pruebas, muchas de ellas innecesarias y sin valor para el paciente, la prescripción de múltiples fármacos, muchas veces innecesarios, que pueden presentar interacciones y efectos secundarios que, probablemente, originarán la prescripción de un nuevo fármaco. Y el paciente se encontrará solo ante esta maraña de profesionales (una media de 13 especialistas si el paciente sufre 5 o más enfermedades crónicas) y sin que nadie se haga responsable de la atención a sus verdaderas necesidades. Y el gasto sanitario se dispara en actos y farmacia que no aportan valor añadido. Somos el país con mayor proporción de gasto farmacéutico (23\% del gasto sanitario total aunque hemos de considerar que este porcentaje está magnificado al ser menor el gasto en capítulo de remuneraciones profesionales) de los países occidentales. Suecia, por ejemplo, con un sistema sanitario y cobertura muy similares a los nuestros, dedica a farmacia solamente un 12\% del total. Los resultados en salud no justifican esta enorme diferencia, solo atribuible al comportamiento del sistema sanitario.

Todos, profesionales y administración, repetimos hasta la saciedad y desde hace varios años, que los pacientes necesitan atención continuada e integrada. ¿Por qué no nos ponemos seriamente a la tarea? En mi opinión, dos son las circunstancias que contribuyen a la inmovilidad. En primer lugar, la humana resistencia al cambio y, en segundo, la falta de liderazgo claro para llevarlo a cabo. En todas las Comunidades Autónomas existen planes estratégicos para este fin pero ¿cuántas los están llevando a cabo de forma rigurosa y estructurada, alineando objetivos, evaluando resultados e incen- 
tivando los logros? Solamente en aquellas entidades locales donde los profesionales se han puesto a liderar el cambio, se han producido verdaderos éxitos en este campo. Pero son pocos y aislados. La situación actual, con la pandemia de las enfermedades crónicas y la adversa coyuntura económica, no permite más dilaciones. Si queremos que nuestro sistema sanitario uno de los mejores del mundo- sobreviva, no podemos quedarnos parados y anclados en nuestra forma de prestar la asistencia. Y no podemos esperar a que el cambio lo impulsen "los otros". Desde hace más de una década, todos los expertos están de acuerdo en que el modelo Chronic Care Model es el más apropiado para la atención a los pacientes crónicos y que la segmentación de los pacientes para prestar diferente tipo de asistencia según sus necesidades, adoptada por la organización sanitaria sin ánimo de lucro Kaiser Permanente, ha demostrado excelentes resultados en salud, satisfacción de los pacientes y profesionales y ahorro en costes. Sin detrimento en el desarrollo de experiencias innovadoras que puedan mejorar ambos modelos, debemos comenzar a aplicarlos en nuestro entorno, con las adaptaciones necesarias a cada lugar. Y, debido a que el segmento más alto de la pirámide de Kaiser, el ocupado por los pacientes crónicos complejos, un 6\% de la población, es el mayor consumidor de recursos, debe ser el primero en ser abordado. Aquí nos encontramos con el primer problema. ¿Cómo estratificar a la población? Los sistemas de información deben permitirlo pero debemos ponernos de acuerdo en todas la Comunidades Autónomas para diseñar un sistema de selección eficaz y común para todas. Un paciente no puede pasar de un segmento a otro según cambie de lugar de residencia. Y ¿qué hacer mientras esto sucede? Creo que los profesionales podemos ir avanzando seleccionado aquellos pacientes que ingresan 20 más veces al año por descompensación de sus patologías crónicas.

Estos pacientes precisan una gestión de caso que no es más (ni menos) que se configure un equipo de profesionales y diseñe una vía o ruta asistencial para ese paciente individual. El equipo básico será, como no puede ser de otra manera, el médico y enfermero de primaria. Este equipo básico debe contar con un equipo de apoyo (internista, enfermero gestor de casos, enfermero de enlace, trabajador social, psicólogo, terapeuta, farmacéutico etc.) que realizarán una valoración integral del paciente, especificarán sus necesidades clínicas, psicológicas o sociales y planificarán todas las actuaciones a realizar para conseguir que el paciente las tenga cubiertas y, además, esté informado de su enfermedad, conozca las señales de alarma ante descompensaciones y forme parte activa de su equipo. Se diseñarán la frecuencia y tipo de contactos que se establecerán con el paciente y "quién hará qué" dentro del equipo, de tal forma que el enfermo no salga nunca del "radar" del sistema socio-sanitario. En el caso de estos pacientes crónicos complejos es indispensable el valor añadido que el internista puede aportar, tanto en la valoración inicial como en la planificación de la ruta clínica, como consultor de referencia ante situaciones previamente determinadas y como responsable o tutor del paciente en caso de que precise alguno de los dispositivos hospitalarios.

Debemos aprender a formar equipos excelentes, hemos de formarnos en las nuevas competencias que esta reorganización del sistema exige y tenemos que constituir alianzas con las entidades comunitarias para combatir con éxito el reto que tenemos planteado. El esfuerzo será importante porque, durante un tiempo, deberemos simultanear la prestación asistencial tradicional con esta nueva orientación, pero tenemos que dar respuesta a nuestros pacientes y a nuestro sistema, sin demora. En la SEMI estamos muy concienciados del problema y, junto a la Sociedad Española de Medicina Familiar y Comunitaria, estamos realizando acciones para impulsar el cambio. EI IV Congreso para la Atención a Pacientes Crónicos es una de ellas. Contamos con el apoyo de muchas otras Sociedades Científicas involucradas en la atención a pacientes crónicos y de las Administraciones. Estoy segura de que, entre todos, seremos capaces de superar el gran desafío al que nos enfrentamos. 